\title{
DIAGNÓSTICO DEL ESTADO DE TRANSFORMADORES DE POTENCIA MEDIANTE EL ANÁLISIS DE GASES DISUELTOS (DGA)
}

Ing. DÍAZ, María Soledad, Ing. SCHMIDT, Erardo $\left(^{*}\right)$

\section{RESUMEN}

El objeto principal del presente trabajo, se enfocó en presentar un panorama general del estado actual de un total de diecinueve (19) Transformadores de Potencia, empleando como herramienta el Análisis de Gases Disueltos (DGA), con base en los registros de análisis cromatográficos, ordenes de mantenimiento preventivo/ correctivo y registro histórico de cada unidad ejecutada durante el período 2014.

Esta prueba se basa en la separación de la muestra en sus distintos compuestos, detectando los gases claves o característicos presentes en el aceite, los cuales contribuyan a identificar la presencia de una falla incipiente.

En el informe, se analizó el contenido de gases disueltos a través de cuatro métodos de interpretación (las relaciones de Rogers, las relaciones de Dornenburg, método Gas Clave y el Triángulo de Duval), además de una serie de criterios de evaluación, los cuales proporcionaron un diagnóstico parcial del estado de las unidades transformadoras. El análisis cromatográfico forma parte de un plan de mantenimiento preventivo.

E1 proceso de análisis comenzó con la recopilación de la documentación pertinente: Informe de análisis cromatográficos y registros de tareas de mantenimiento; se estableció un diagnóstico de cada unidad en base al análisis y a los criterios mencionados anteriormente, finalmente se realizó una evaluación de los resultados obtenidos y se presentaron las conclusiones y recomendaciones al respecto.

PALABRAS CLAVE: Análisis de Gases Disueltos, Cromatografía; Transformadores de Potencia, Diagnóstico

\section{DIAGNÓSTIGO INTEGRADO} DE TRANSFORMADORES DE POTENCIA

Dentro del sistema de aislamiento se encuentra el aceite dieléctrico el cual además de funcionar como barrera dieléctrica entre los componentes del transformador y ser refrigerante, brinda gran información del estado de funcionamiento del transformador.

Con el deterioro de los materiales aislan- 
tes se forman diferentes tipos de gases que se van diluyendo en el aceite aislante y que pueden evidenciar la presencia de una falla dentro del transformador, a través de una buena interpretación se puede llegar a detectar fallas incipientes y actuar en forma correctiva, evitando así la generación de fallas mayores y la interrupción del suministro eléctrico.

"Los materiales aislantes en transformadores se descomponen liberando gases dentro de la cuba; la distribución de éstos gases puede estar relacionada con el tipo de avería del suministro, y la tasa de generación de gas puede indicar su severidad". (SARRIA ARIAS et al., 2014).

El diagnóstico mediante el Análisis de Gases Disueltos (DGA), se emplea desde el año 1960 para la detección de averías incipientes en Transformadores de Potencia.

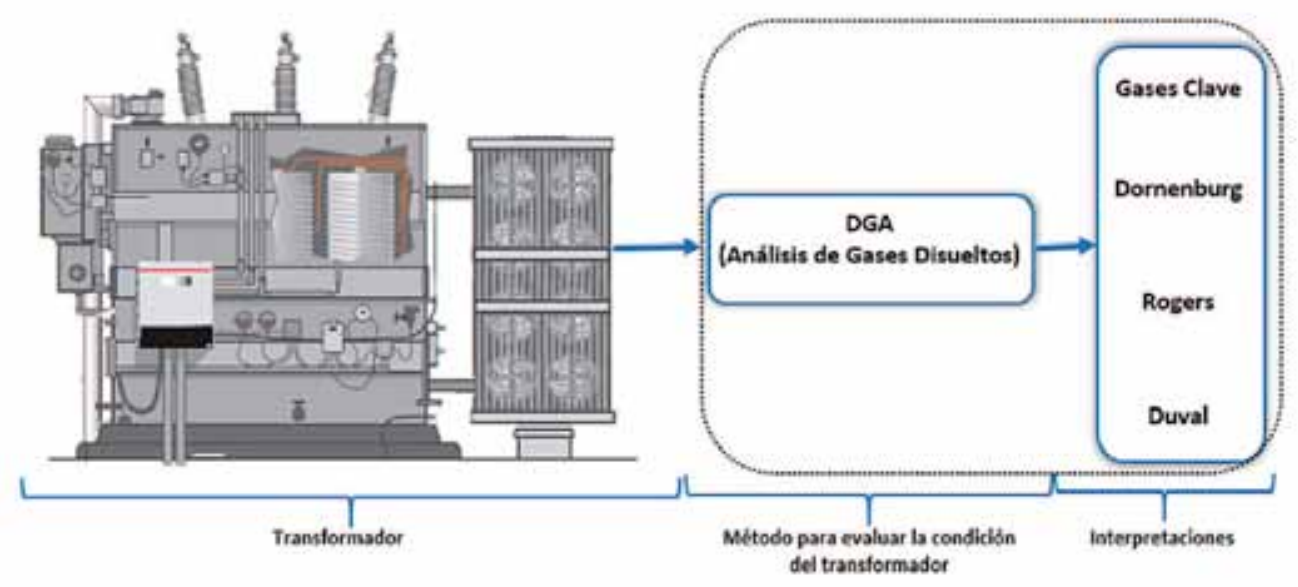

Ilustración 1. Diagrama conceptual de la aplicabilidad del DGA. Fuente: SARRIA ARIAS et al., 2014.

Mencionado lo anterior, es necesario determinar las condiciones en que se encuentra el aceite aislante mediante un análisis cromatográfico, el cual debe estar contemplado en el programa de mantenimiento preventivo anual del transformador; con el objetivo de recolectar información acerca de la cantidad de gases disueltos y análisis físico químicos. Para las unidades observadas, estos análisis son efectuados por un laboratorio externo, con la periodicidad anteriormente mencionada, excepto cuando se detecta una condición anormal.

Con el objetivo de obtener un análisis integral, se analizaron los parámetros de desempeño de los diecinueve (19) Transformadores de Potencia, a partir del año 2010; haciendo hincapié en los resultados del último informe de análisis cromatográfico del año 2014 de cada unidad transformadora.

Cada unidad transformadora se evaluó y analizó aplicando los métodos de Análisis de Gases Disueltos (DGA) por los siguientes criterios: Diagnóstico Dornenburg, Método Roger, Triángulo de Duval y Gas Clave, además de una serie de criterios, todos de amplia utilización para la estimación de posibles fallas en el análisis de Transformadores de Potencia. En la Tabla No5, se muestran los criterios de relación para las diecinueve unidades transformadoras analizadas. A 
continuación se presenta una breve descripción de los métodos y criterios utilizados:

- Diagnóstico Dornenburg: Se basa en cuatro relaciones entre concentracio- nes de gases R1:CH4/H2; R2:C2H2/ $\mathrm{C} 2 \mathrm{H} 4, \quad \mathrm{R} 3: \mathrm{C} 2 \mathrm{H} 2 / \mathrm{CH} 4, \quad \mathrm{R} 4: \mathrm{C} 2 \mathrm{H} 6 /$ $\mathrm{C} 2 \mathrm{H} 2$, presenta la desventaja que solo se puede aplicar si existe una cantidad adecuada de gases disueltos en el aceite.

\begin{tabular}{|c|c|c|c|c|}
\hline \multirow{2}{*}{ TIPO DE FALLA } & \multicolumn{4}{|c|}{ RELACIONES } \\
\cline { 2 - 5 } & R1 & R2 & R3 & R4 \\
\hline Descomposición Térmica & $>1.0$ & $<0.75$ & $<0.3$ & $>0.4$ \\
\hline $\begin{array}{c}\text { Descarga Parcial de Baja } \\
\text { Intensidad (Efecto Corona) }\end{array}$ & $<0.1$ & $\begin{array}{c}\text { No } \\
\text { Significativa }\end{array}$ & $<0.3$ & $>0.4$ \\
\hline $\begin{array}{c}\text { Descarga Parcial de Alta } \\
\text { Intensidad (Arco) }\end{array}$ & $>0.1<1.0$ & $>0.75$ & $>0.3$ & $<0.4$ \\
\hline
\end{tabular}

Tabla 1. Interpretación de la Falla mediante el Diagnóstico de Dornenburg. Fuente: Elaboración propia.

- Método Roger: Este método introduce una nueva relación entre los gases Etileno $(\mathrm{C} 2 \mathrm{H} 4)$ y Acetileno $(\mathrm{C} 2 \mathrm{H} 2)$, los cuales requieren de una temperatura elevada para generarse, a su vez elimina las relaciones entre Etano (C2H6) y Metano ( $\mathrm{CH} 4)$, debido a que considera que las mismas no contribuyen en la identificación de la falla.

\begin{tabular}{|c|c|c|c|c|}
\hline \multicolumn{2}{|r|}{ RANGO DE RELACIONES } & $\begin{array}{c}\mathrm{R} 1 \\
(\mathrm{CH} 4 / \mathrm{H} 2)\end{array}$ & $\begin{array}{c}\mathrm{R} 2 \\
(\mathrm{C} 2 \mathrm{H} 2 / \mathrm{C} 2 \mathrm{H} 4)\end{array}$ & $\begin{array}{c}\mathrm{R3} \\
(\mathrm{C} 2 \mathrm{H} 4 / \mathrm{C} 2 \mathrm{H} 6)\end{array}$ \\
\hline \multicolumn{2}{|r|}{$<0.1$} & 1 & 0 & 0 \\
\hline & $0.1-1.0$ & 0 & 1 & 0 \\
\hline & $1.0-3.0$ & 2 & 1 & 1 \\
\hline & $>3.0$ & 2 & 2 & 2 \\
\hline CASO & TIPO DE FALLA & \multicolumn{3}{|c|}{ CÓDIGO } \\
\hline 1 & Descargas Parciales de Baja Intensidad & 1 & 1 & 0 \\
\hline 2 & Descargas Parciales de Alta Intensidad & 1 & 1 & 0 \\
\hline 3 & $\begin{array}{c}\text { Descargas de baja energía, chispeo y } \\
\text { arco }\end{array}$ & 0 & $1-2$ & $1-2$ \\
\hline 4 & Descargas de alta energía arco & 0 & 1 & 2 \\
\hline 5 & $\begin{array}{l}\text { Falla Térmica por Temp. Inferiores a } \\
\qquad 150^{\circ} \mathrm{C}\end{array}$ & 0 & 0 & 1 \\
\hline 6 & $\begin{array}{c}\text { Falla Térmica por Temp. Entre } 150^{\circ} \mathrm{C} \\
\text { y } 300^{\circ} \mathrm{C}\end{array}$ & 2 & 0 & 0 \\
\hline 7 & $\begin{array}{c}\text { Falla Térmica por Temp. Entre } 300^{\circ} \mathrm{C} \\
\text { y } 700^{\circ} \mathrm{C}\end{array}$ & 2 & 0 & 1 \\
\hline 8 & $\begin{array}{l}\text { Falla Térmica por Temp. Superior a } \\
\qquad 700^{\circ} \mathrm{C}\end{array}$ & 2 & 0 & 2 \\
\hline
\end{tabular}

Tabla 2. Interpretación de falla mediante Método Rogers. Fuente: Elaboración propia.

- Triángulo de Duval: Duval emplea un triángulo equilátero compuesto por tres vértices: $\% \mathrm{CH} 4 ; \% \mathrm{C} 2 \mathrm{H} 2$ y $\% \mathrm{C} 2 \mathrm{H} 2$. Esta técnica propone un sistema de ecuaciones para el cálculo de las concentraciones en partes por millón.
$\%$ C2H4 $=\left[\frac{100 y}{x+y+z}|\% C 2 H 2=| \frac{100 x}{x+y+z}|\% C H 4=| \frac{100 z}{x+y+z} \mid\right.$

Dónde: $x$ es la concentración de Acetileno $(\mathrm{C} 2 \mathrm{H} 2)$ en ppm; $y$ es la concentración de Etileno (C2H4) en ppm y $z$ es la concentración de Metano (CH4) en ppm. 


\begin{tabular}{|c|c|}
\hline ZONA & TIPO DE FALLA \\
\hline D1 & Descarga de Baja Energía \\
\hline D2 & Descarga de Alta Energía \\
\hline DT & $\begin{array}{l}\text { Falla térmica y eléctrica en } \\
\text { conjunto }\end{array}$ \\
\hline $\mathrm{T} 1$ & Falla térmica por Temp. $<300^{\circ} \mathrm{C}$ \\
\hline $\mathrm{T} 2$ & $\begin{array}{l}\text { Falla térmica por Temp. entre } \\
\qquad 300^{\circ} \mathrm{C} \text { y } 700^{\circ} \mathrm{C}\end{array}$ \\
\hline T3 & Falla térmica por Temp. $>700^{\circ} \mathrm{C}$ \\
\hline PD & Descarga Parcial \\
\hline
\end{tabular}

Tabla 3. Interpretación de las zonas del Triángulo de Duval. Fuente: Elaboración propia.

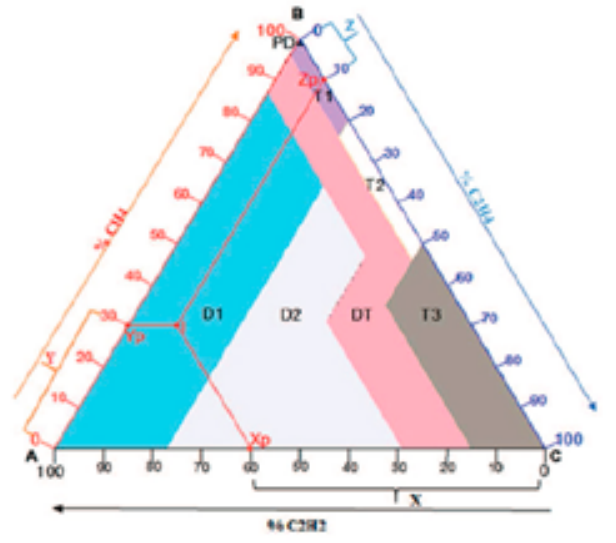

Gráfico 1. Ubicación de la falla en el Triángulo de Duval. Fuente: SARRIA ARIAS et al., 2014.

- Gas Clave: Esta técnica de- de los gases individuales en vez de tecta la falla mediante la medición calcular las proporciones del gas.

\begin{tabular}{|c|c|c|}
\hline TIPO DE FALLA & GAS CLAVE & $\%$ GAS PRESENTE \\
\hline $\begin{array}{l}\text { Descarga Parcial de Alta } \\
\text { Intensidad (Arco) }\end{array}$ & $\begin{array}{l}\text { Acetileno } \\
(\mathrm{C} 2 \mathrm{H} 2)\end{array}$ & $\begin{array}{c}\mathrm{CO}:<0.1 \% \\
\mathrm{H} 2: 60 \% \\
\mathrm{CH} 4: 5 \% \\
\mathrm{C} 2 \mathrm{H} 6: 1.6 \% \\
\mathrm{C} 2 \mathrm{H} 4: 3.3 \% \\
\mathrm{C} 2 \mathrm{H} 2: 30 \%\end{array}$ \\
\hline $\begin{array}{l}\text { Descarga Parcial de Baja } \\
\text { Intensidad (Efecto Corona) }\end{array}$ & $\begin{array}{l}\text { Hidrógeno } \\
\text { (H2) }\end{array}$ & $\begin{array}{c}\text { CO: } 0.2 \% \\
\text { H2: } 86 \% \\
\text { CH4: } 13 \% \\
\text { C2H6: } 0.5 \% \\
\text { C2H } 4: 0.2 \% \\
\text { C2 } 2 \text { 2: } 0.1 \%\end{array}$ \\
\hline $\begin{array}{l}\text { Sobrecalentamiento del } \\
\text { Aceite Aislante }\end{array}$ & $\begin{array}{l}\text { Etileno } \\
\text { (C2H4) }\end{array}$ & $\begin{array}{c}\mathrm{CO}:<0.01 \% \\
\mathrm{H} 2: 2 \% \\
\text { CH4: } 16 \% \\
\text { C2H6: } 17 \% \\
\text { C2 } 2 \mathrm{H} 4: 63 \% \\
\text { C2H2: } 2 \%\end{array}$ \\
\hline $\begin{array}{l}\text { Sobrecalentamiento de la } \\
\text { Celulosa Aislante }\end{array}$ & $\begin{array}{l}\text { Monóxido de Carbono } \\
\text { (CO) }\end{array}$ & $\begin{array}{c}\mathrm{CO}: 92 \% \\
\mathrm{H} 2: 6.7 \% \\
\text { CH4: } 1.2 \% \\
\mathrm{C} 2 \mathrm{H} 6:<0.01 \% \\
\mathrm{C} 2 \mathrm{H} 4:<0.01 \% \\
\mathrm{C} 2 \mathrm{H} 2: 0.01 \%\end{array}$ \\
\hline
\end{tabular}

Tabla 4. Interpretación de falla mediante método Gas Clave. Fuente: Adaptado de SARRIA ARIAS et al., 2014.

El aceite genera gases como aceti- ra CO y CO2 (IEC 60599, 2007), "la leno (C2H2), hidrógeno (H2), metano vida del transformador es la vida del papel", (CH4), etileno (C2H4) y etano (C2H6), este tipo de aislamiento está considerado mientras que la celulosa o papel gene- como el eslabón más débil en la cadena. 
Los gases de falla se pueden dividir en tres grandes categorías: Descarga Corona o Descarga Parcial; Calentamiento térmico y Arqueo, las mismas difieren en la intensidad de energía, la más severa ocurre con el Arqueo.

\section{Clasificación de los Gases:}

- Hidrocarburos: Metano (CH4); Etano (C2H6); Etileno (C2H4); Acetileno (C2H2) e Hidrógeno (H2).

- Óxido de Carbono: Monóxido de Carbono (CO) y Dióxido de Carbono (CO2).

- Gases Libres: Nitrógeno (N2) y Oxígeno (O2).

- Relaciones entre Monóxido de Carbono (CO) y Dióxido de Carbono (CO2): En los siguientes rangos $0.3 \leq \mathrm{CO} /$ $\mathrm{CO} 2 \leq 0.7$ y $3 \leq \mathrm{CO} 2 / \mathrm{CO} \leq 11$, las mismas determinan si existen fallas que involucran a la celulosa, ya que se tratan de los principales componentes de su combustión. El estado del aislamiento sólido se refleja en la relación monóxido-dióxido $(\mathrm{CO} 2 / \mathrm{CO})$, el contenido de $\mathrm{CO} 2$ es alto con respecto al $\mathrm{CO}$, esto indica una falta de enfriamiento y sobrecalentamiento general en las unidades. La Oxidación y calentamiento térmico del aceite causa producción de $\mathrm{CO}$ y $\mathrm{CO} 2$; un valor de relación de $\mathrm{CO} 2 / \mathrm{CO}$ superior a 11 indica la posibilidad de existencia de una falla del tipo térmica.

- Total de Gases Combustibles Disueltos (TDCG): Según la norma IEC 60599 (2007), el límite de aceptación del TDCG en el aceite de un Transformador de Potencia debe estar entre 560ppm y $1250 \mathrm{ppm}$, mientras que la norma IEEE C57.104 (2008) plantea que este valor puede ser máximo 700ppm.

- Relación Etano (C2H6)/Etileno (C2H4): $\mathrm{Si}$ aparece $\mathrm{C} 2 \mathrm{H} 4$ sin $\mathrm{C} 2 \mathrm{H} 2$ y el diagnóstico que se da es "punto caliente en el aislamiento sólido", lo que es cierto, pero se ignora la existencia de descargas parciales en el aislamiento sólido. Si en este caso la relación $\mathrm{CO} / \mathrm{CO} 2>0,1$, debe suponerse la superposición de estos dos efectos; igualmente una relación $\mathrm{C} 2 \mathrm{H} 6 / \mathrm{C} 2 \mathrm{H} 4>1$ indica siempre descargas parciales.

- Contenido de Agua (H2O): E1 agua disuelta afecta las propiedades dieléctricas del aceite. Un alto contenido de agua acelera la degradación química del papel aislante. Las Normas establecen un valor en el contenido de $\mathrm{H} 2 \mathrm{O}$ por debajo de las 20 ppm, a su vez indican que un valor a partir de 10 ppm se considera observable.

- El contenido general de gases de aire (GA): Representa la relación existente entre los gases libres (O2 y N2), se debe encontrar por debajo del valor de $4 \%$ establecido por la norma de uso general para transformadores herméticos que se encuentren en explotación.

\section{CONCLUSIONES Y RECOMEN- DACIONES}

- Considerando los criterios que relacionan $\mathrm{CO}$ y CO2, todos los Transformadores de Potencia analizados, se encuentran fuera del rango establecido para la relación $\mathrm{CO} 2 / \mathrm{CO}$, lo que indica un calentamiento térmico a baja temperatura. 


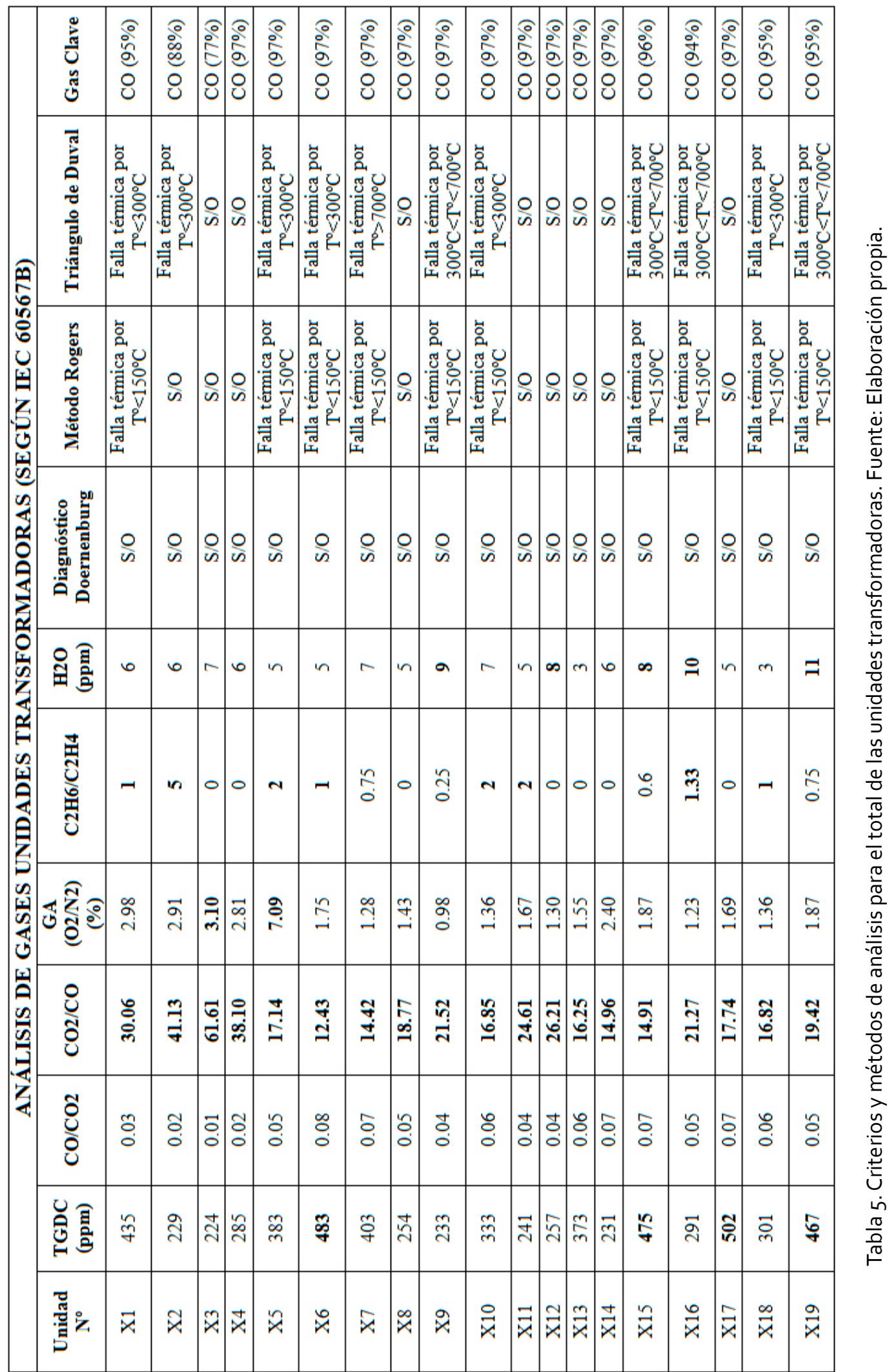


- En cuanto al criterio del Total de Gases Combustibles Disueltos (TGDC), todas las unidades transformadoras cumplen con la condición establecida por la Norma IEC 60599, las unidades X6; X15, X17 y X19, presentan valores entre 467 y 502 ppm, los cuales pueden ser considerados relativamente altos por la Norma IEEE C57.104 (2008).

- $\quad$ Las unidades X1; X2; X5; X6; X10; $\mathrm{X} 11 ; \mathrm{X} 16$ y X18, poseen una relación de $\mathrm{C} 2 \mathrm{H} 6 / \mathrm{C} 2 \mathrm{H} 4>1$, la cual indica la presencia de pequeñas descargas parciales en el aislamiento sólido, las cuales se superponen con una falla asociada al envejecimiento térmico normal.

- El contenido general de gases de aire (GA) la cual representa la relación existente entre los gases libres (O2 y N2), se debe encontrar por debajo del valor de $4 \%$ establecido por la norma de uso general para transformadores herméticos que se encuentren en explotación; la mayoría de las unidades cumple con dicha condición, salvo la unidad X5, la cual presenta un valor de GA del 7.09\%, por lo que se recomienda efectuar una desgasificación en dicho transformador.

- $\quad$ El contenido de agua (H2O), representa otro parámetro de análisis, su presencia puede afectar las propiedades dieléctricas del aceite, provoca una disminución de

\section{REFERENCIAS BIBLIOGRÁFICAS}

1. ACEVEDO, J. (2009). "Diagnóstico de condición de equipos de subestaciones eléctricas de Alta Tensión”. Congreso Internacional en Alta Tensión y aisla- la rigidez dieléctrica y de la resistividad y un aumento del factor de disipación dieléctrica. Un alto contenido de agua acelera la degradación química del papel aislante. Las unidades X9; X12; X15; X16 y X19 presentan valores de contenido de agua observables.

- De acuerdo a los Criterios de Diagnóstico por DGA, las Unidades X3; X4; X8; X11; X12; X13; X14 y X17, se encuentran funcionando normalmente. Los valores de monóxido y dióxido de carbono corresponden a un envejecimiento normal del papel aislante (celulosa).

- $\quad$ Las Unidades X1; X2; X5; X6; X7; X9; X10; X15; X16; X18 y X19 presentan valores de relaciones observables y los diferentes criterios de diagnóstico indican la posible existencia de fallas de origen térmico, las cuales comprometen al aislamiento sólido (papel aislante). Se recomienda efectuar dos análisis cromatográficos por año.

- Es prematuro concluir con un pronóstico y diagnóstico del estado operativo real de cada transformador, debido a que es necesaria la obtención de más datos a través de otros controles y/o ensayos (Termografía infrarroja, Análisis de Respuesta en Frecuencia, Descargas Parciales, etc.), que permitan aportan evidencia a favor de los criterios anteriormente mencionados.

miento eléctrico. Medellín, Colombia.

2. AGUIRRE GARCÍA, et al. (2012). "Diagnóstico estado actual Transformadores Principales y Autotransformadores en la 
CHY”. X Seminario del Sector Eléctrico Paraguayo - CIGRÉ. Asunción, Paraguay.

3. ARTERO, J. (2009). "Mantenimiento Moderno en Transformadores de Potencia”.Empresa Transmisora de El Salvador, ETESAL S.A. XIII Encuentro Regional Iberoamericano de CIGRÉ. Puerto Iguazú, Argentina.

4. CARRERA GÓMEZ, A. (2013). “Análisis de gases disueltos en aceite para mantenimiento preventivo a transformadores de potencia mediante la implementación de software". Instituto Politécnico Nacional. Unidad Zacatenco, México.

5. IEC 60599. (2007). "Mineral Oil - Impregnated electrical equipment in service - Guide to the interpretation of disolved and free gases analysis". International
Electrotechnical Commission (IEC).

6. IEEE C57.104 (2008). "Guide for the Interpretation of Gases Generated in OilImmersed Transformers". Institute of Electrical and Electronics Engineers (IEEE).

7. REYES PÉREZ, I. (2010). "Procedimiento para realizar e interpretar la prueba cromatográfica de gases en Transformadores de Potencia”. Universidad nacional Autónoma de México. Facultad de Ingeniería. Distrito Federal, México.

8. SORRIA ARIAS, J. et al. (2014). "Estado del arte del análisis de gases disueltos en Transformadores de Potencia”. Universidad Distrital "Francisco José Caldas". Revista Facultad de Ingeniería. Enero-Junio 2014. Vol.24, No36, pp. 105-122. ISSN 01211129. Bogotá-Cundinamarca, Colombia. 